\title{
Authorized Auxiliary Medicinal Product
}

National Cancer Institute

\section{Source}

National Cancer Institute. Authorized Auxiliary Medicinal Product. NCI Thesaurus. Code C156618.

A medicinal product with marketing authorization that is used in a clinical trial for purposes other than as the investigational agent. 